hep-th/0012084

FSU TPI 08/00

\title{
PRECANONICAL QUANTIZATION AND THE SCHRÖDINGER WAVE FUNCTIONAL
}

\author{
Igor V. Kanatchikov ${ }^{a, b, *, \dagger}$ \\ a Institute of Theoretical Physics, Friedrich Schiller University of Jena \\ Max-Wien-Platz 1, D-07743 Jena, Germany \\ ${ }^{b}$ Laboratory of Analytical Mechanics and Field Theory \\ Institute of Fundamental Technological Research \\ Polish Academy of Sciences, Warsaw 00-049, Poland \\ final version, April 052001 \\ accepted for publication in Phys. Lett. A
}

\begin{abstract}
A relation between the Schrödinger wave functional and the Clifford-valued wave function which appears in what we call precanonical quantization of fields and fulfils a Dirac-like generalized covariant Schrödinger equation on the space of field and space-time variables is discussed. The Schrödinger wave functional is argued to be the trace of the positive frequency part of the continual product over all spatial points of the values of the aforementioned wave function restricted to a Cauchy surface. The standard functional differential Schrödinger equation is derived as a consequence of the Dirac-like covariant Schrödinger equation.
\end{abstract}

\section{Introduction}

The precanonical approach to field quantization [1] 9] is based on a generalization of the Hamiltonian formulation from mechanics to field theory which requires no space-time decomposition and manages to incorporate the field dynamics into a finite dimensional covariant analogue of the phase space. The generalizations of this kind have been long known in the calculus of variations [10 [17]. Being based on a covariant analogue of the Legendre transformation and the Hamilton first order canonical equations but still independent of the picture of fields as infinite dimensional systems evolving with time, these generalizations are in a sense intermediate between the Lagrangian and the standard canonical Hamiltonian formulation, hence the name "precanonical." The simplest and the basic for the present paper example is the so-called De Donder-Weyl (DW) formulation [10 13]: given a Lagrangian density $L\left(y^{a}, y_{\mu}^{a}, x^{\nu}\right)$, a function of field

\footnotetext{
${ }^{*}$ E-mail: kai@tpi.uni-jena.de, kai@fuw.edu.pl, URL: http://www.tpi.uni-jena.de/kai.

${ }^{\dagger}$ On leave from Tallinn Technical University, 19086 Tallinn, Estonia.
} 
variables $y^{a}$, their space-time derivatives (first jets) $y_{\mu}^{a}$, and space-time variables $x^{\mu}$, one introduces new Hamiltonian-like variables: $p_{a}^{\mu}:=\partial L / \partial y_{\mu}^{a}$ (polymomenta) and $H=$ $H\left(y^{a}, p_{a}^{\mu}, x^{\nu}\right):=y_{\mu}^{a} p_{a}^{\mu}-L($ the DW Hamiltonian function), and writes the field equations in the appealing Hamiltonian-like first order form [10 13]

$$
\partial_{\mu} y^{a}(x)=\frac{\partial H}{\partial p_{a}^{\mu}}, \quad \partial_{\mu} p_{a}^{\mu}(x)=-\frac{\partial H}{\partial y^{a}},
$$

provided the Lagrangian density is not singular in the sense that $\operatorname{det}\left(\left\|\frac{\partial^{2} L}{\partial y_{\mu}^{a} \partial y_{\nu}^{b}}\right\|\right) \neq 0$.

In this formulation fields are viewed as sections $y^{a}(x)$ in a bundle of the field variables over the space time, a finite dimensional covariant configuration bundle with the local coordinates $\left(y^{a}, x^{\mu}\right)$. In a sense the formulation (1.1) can be considered as a "multi-temporal" generalization of the Hamiltonian formulation to field theory since the space and time variables are present in (1.1) on equal footing as analogues of the time variable in mechanics. As a byproduct, this treatment makes the formulation applicable even when the space-time is not globally hyperbolic. The analogue of the phase space involved in (1.1) is a finite dimensional space of variables $\left(y^{a}, p_{a}^{\mu}, x^{\mu}\right)$ called the (extended) polymomentum phase space.

The manifest covariance and the "finite dimensionality" in the aforementioned sense are the common features of a whole class of Hamiltonian-like formulations known from the theory of Lepagean equivalents in the calculus of variation [15]20]. The above mentioned De Donder-Weyl formulation represents the most straightforward covariant generalization of the Hamiltonian formulation in mechanics. Other Lepagean theories are based on more sophisticated definitions of polymomenta and the covariant Hamiltonian functions $H$ and still remain little known.

The appropriate Poisson bracket operation for the precanonical DW formulation (1.1) has been introduced in 21 23] (see also 24 27] for recent attempts of generalizations). The Poisson brackets are defined on appropriate differential forms, which play the role of dynamical variables, and lead to a Gerstenhaber algebra structure generalizing the Poisson algebra of observables in classical mechanics. The structures of the Hamiltonian formalism in mechanics are reproduced in the case of $(0+1)$-dimensional "field theory". The Poisson bracket on forms enables us to specify the notion of (pre)canonically conjugate field and polymomentum variables and to write the DW Hamiltonian equations (1.1) in Poisson bracket formulation in which the bracket with $H$ is related to the operation of the total exterior differentiation, the latter generalizing the total time derivative in the Poisson bracket formulation of the equations of motion in mechanics (see [2, 21 23] for more details).

Quantization based on these structures leads to the idea of a partial differential generalized Schrödinger equation for the wave function on the covariant configuration space: $\Psi=\Psi\left(y^{a}, x^{\mu}\right)$. The following Dirac-like equation has been put forward as a "multi-temporal" covariant generalization of the Schrödinger equation from quantum mechanics to field theory [1] 3]:

$$
i \hbar \varkappa \gamma^{\mu} \partial_{\mu} \Psi=\widehat{H} \Psi
$$


where $\widehat{H}$ denotes the operator of the DW Hamiltonian, and $\varkappa$ is a (large) constant of the dimension length ${ }^{-3}$ (in $n=4$ space-time dimensions) which is interpreted as the ultraviolet cutoff of the volume of $\mathbf{k}$-space (or an inverse characteristic "minimal volume" in $\mathrm{x}$-space); and $\Psi=\Psi\left(y^{a}, x^{\mu}\right)$ is a Clifford- (or spinor-) valued wave function of a quantized field. Note that $\varkappa$ appears on purely dimensional grounds.

The probabilistic interpretation of the wave function $\Psi\left(y^{a}, x^{\mu}\right)$ follows from the positive definiteness of $\bar{\Psi} \gamma^{0} \Psi$ for the Dirac spinor valued $\Psi$-s and from the conservation law

$$
\partial_{\mu} \int d y \bar{\Psi} \gamma^{\mu} \Psi=0
$$

which can be derived from (1.2). One can interpret $\Psi\left(y^{a}, x^{\mu}\right)$ as the probability amplitude of finding the field value $y^{a}$ in (the vicinity of) the space-time point $x^{\mu}$. Obviously, the description of quantized fields in terms of this wave function is fundamentally different from any standard description of quantum fields.

The choice of the wave equation (1.2) is based on the observations that (i) it fulfils an analogue of the Ehrenfest theorem in the sense that the DW Hamiltonian equations (1.1) can be derived from (1.2) as the equations for the expectation values of properly chosen operators [2, 3], and that (ii) in the classical limit (1.2) can be reduced to the Hamilton-Jacobi equation of the De Donder-Weyl theory (see Sect. 3 and [10 [14]) by means of the ansatz $\Psi=R e^{i S^{\mu} \gamma_{\mu} / \hbar \varkappa}$ [1, 3], where $S^{\mu}$ are the eikonal functions of the DW Hamilton-Jacobi theory (see Sect. 3).

Quantization of a small Lie subalgebra of canonically conjugate field and polymomentum variables (given by proper differential forms) [3, 21] suggests the following operator realization of polymomenta [2, 3]

$$
\hat{p}_{a}^{\mu}=-i \hbar \varkappa \gamma^{\mu} \frac{\partial}{\partial y^{a}}
$$

where the constant $\varkappa$ appears again on dimensional grounds.

It is noteworthy that the "multi-temporal" quantum theoretic formalism which appears here as a way of description of quantized fields can be viewed as a hypercomplex extension of the formalism of quantum mechanics in which the wave functions and operators take values in the Clifford algebra of the underlying space-time manifold. The quantum mechanics appears then as a particular case corresponding to a $(0+1)$ dimensional field theory, when the corresponding Clifford algebra of $(0+1)$-dimensional space-time is just the algebra of the complex numbers.

The elements of precanonical quantization as outlined above, of course, differ significantly from any presently known description of quantum fields. Their relation to the elements of the commonly practiced quantum field theory are far from being immediately obvious. This is the purpose of the present paper to clarify at least one aspect of this relation, namely an interplay between the wave function $\Psi\left(y^{a}, x^{\mu}\right)$ and the Schrödinger wave functional, and the interrelation between the Dirac-like wave equation (1.2) and the functional differential Schrödinger equation. The possibility of establishing of such a connection seems to be an important argument in favour of the wave equation (1.2) and precanonical quantization. 
In Section 2 we recall the elements of canonical and precanonical quantization of the scalar field which are used in what follows. An interplay between the functional differential Hamilton-Jacobi equation of the canonical formalism and the partial differential Hamilton-Jacobi equation of the precanonical De Donder-Weyl theory is discussed in Section 3. This result is extended to the quantum level in Section 4 where we show how the functional differential Schödinger equation and the Schödinger wave functional are derived from the partial differential Dirac-like generalized Schödinger equation of the precanonical approach and the corresponding Clifford-valued wave function. Concluding remarks are presented in Section 5.

\section{Two Quantizations of the Scalar Field}

In this section we outline canonical and precanonical quantization of the free scalar field given by the Lagrangian density (henceforth we set $\hbar=1$ )

$$
L=\frac{1}{2} \partial_{\mu} y \partial^{\mu} y-\frac{1}{2} m^{2} y^{2} .
$$

Our main purpose is to present a background on both approaches necessary for the discussion of an interplay between them in the subsequent sections.

\subsection{Canonical quantization}

Let us recall some basic elements of canonical quantization of the scalar field in the functional Schrödinger representation 30 32]. The canonical momentum is defined as $\pi(\mathbf{x})=\partial L / \partial\left(\partial_{t} y(\mathbf{x})\right)$ and the canonical Hamiltonian functional, which is a functional of $y(\mathbf{x})$ and $\pi(\mathbf{x})$, as $\mathbf{H}:=\int d \mathbf{x}\left(\partial_{t} y(\mathbf{x}) \pi(\mathbf{x})-L\right)$. From the Lagrangian (2.1) one obtains: $\pi(\mathbf{x})=\partial_{t} y(\mathbf{x})$ and

$$
\mathbf{H}=\frac{1}{2} \int d \mathbf{x}\left(\pi^{2}(\mathbf{x})+\left(\partial_{i} y(\mathbf{x})\right)^{2}+m^{2} y^{2}(\mathbf{x})\right)
$$

Quantization of the canonical Poisson brackets leads to the realization

$$
\hat{\pi}(\mathbf{x})=-i \frac{\delta}{\delta y(\mathbf{x})}
$$

in the functional Scrödinger representation when the quantum states are described by a time dependent functional of field configurations $y(\mathbf{x}): \Psi_{S}=\Psi_{S}([y(\mathbf{x})], t)$ which fulfils the functional Schrödinger equation

$$
i \partial_{t} \boldsymbol{\Psi}_{S}=\widehat{\mathbf{H}}_{S} \boldsymbol{\Psi}_{S},
$$

\footnotetext{
${ }^{1}$ Here and in what follows the bold capital letters are reserved to designate functionals; $\mathbf{x}=x^{i}$ denote the spatial components of a four-vector $x^{\mu}:=\left(x^{i}, x^{t}\right)$.
} 
where the functional Hamiltonian operator for the scalar field has the form

$$
\widehat{\mathbf{H}}_{S}=\frac{1}{2} \int d \mathbf{x}\left(-\frac{\delta^{2}}{\delta y^{2}(\mathbf{x})}+\left(\partial_{i} y(\mathbf{x})\right)^{2}+m^{2} y^{2}(\mathbf{x})\right) .
$$

The ground (vacuum) state wave functional of this Hamiltonian operator can be represented in terms of the Fourier components of $y(\mathbf{x})=\int \frac{d \mathbf{k}}{(2 \pi)^{3}} y(\mathbf{k}) e^{i \mathbf{k x}}$ as follows:

$$
\boldsymbol{\Psi}_{\text {Svac }}=\exp \left(-\frac{1}{2} \int \frac{d \mathbf{k}}{(2 \pi)^{3}} \omega_{\mathbf{k}} y(-\mathbf{k}) y(\mathbf{k})\right)
$$

and it corresponds to the divergent energy eigenvalue

$$
E_{\mathrm{vac}}=\frac{1}{2} \int d \mathbf{x} \int \frac{d \mathbf{k}}{(2 \pi)^{3}} \omega_{\mathbf{k}}
$$

The excited states are known to correspond to the multi-particle (Fock) states which can be generated by the iterated action of the creation operator

$$
a^{\dagger}(\mathbf{k})=\frac{1}{\sqrt{2}} \int d \mathbf{x} e^{-i \mathbf{k x}}\left(-\frac{\delta}{\delta y(\mathbf{x})}+\omega_{\mathbf{k}} y(\mathbf{x})\right)
$$

on the vacuum state wave functional (2.6).

\subsection{Precanonical quantization}

Now, let us quantize the scalar field theory using the precanonical procedure outlined in the Introduction. The Lagrangian density (2.1) gives rise to the polymomenta $p^{\mu}=\partial^{\mu} y$ and the DW Hamiltonian function

$$
H\left(y, p^{\mu}\right)=\frac{1}{2} p^{\mu} p_{\mu}+\frac{1}{2} m^{2} y^{2} .
$$

Precanonical quantizaton leads to the realization of the operators of polymomenta in the form

$$
\hat{p}^{\mu}=-i \varkappa \gamma^{\mu} \partial_{y}
$$

as in (1.4), and to the following operator of the DW Hamiltonian [1] [3]

$$
\widehat{H}:=-\frac{1}{2} \varkappa^{2} \partial_{y y}^{2}+\frac{1}{2} m^{2} y^{2} .
$$

In our previous papers [1, 3] we presented a solution of the generalized Schrödinger equation (1.2) with the DW Hamiltonian operator (2.11) in the basis where the latter is diagonal. However, the fact that there is no physical interpretation of $H$ on the classical level implies that this basis is not physical. One should diagonalize an operator representing a physical quantity, such as the energy. 
Obviously, one can rewrite (1.2) in the space-time decomposed form

$$
i \partial_{t} \Psi=-i \alpha^{i} \partial_{i} \Psi+\beta \frac{1}{\varkappa} \widehat{H} \Psi=: \widehat{\mathcal{E}} \Psi
$$

and interpret the operator $\widehat{\mathcal{E}}$ defined in the right hand side as the energy operator, as it represents the time evolution.

Equation (2.12) is best solved in the Fourier space where it takes the form

$$
k^{t} \Psi(y, \mathbf{k})=\left(-\alpha^{i} \mathbf{k}_{i}+\beta \frac{1}{\varkappa} \widehat{H}\right) \Psi(y, \mathbf{k}),
$$

where $k^{\mu}:=\left(\mathbf{k}^{i}, k^{t}\right)$. Taking into account that $m^{2}$ in $(2.11)$ can be written as $m^{2}=$ : $\left(q_{\mu} \gamma^{\mu}\right)^{2}$ for some $q^{\mu}=\left(\mathbf{q}, \omega_{\mathbf{q}}\right), \omega_{\mathbf{q}}:=\sqrt{m^{2}+\mathbf{q}^{2}}$, one can find the ground state $(N=0)$ solution (up to a normalisation factor)

$$
\Psi_{0}(y, \mathbf{q})=e^{-\frac{1}{2 \varkappa} q_{\mu} \gamma^{\mu} y^{2}}
$$

which corresponds to the eigenvalues $k_{0}^{t}=\frac{1}{2} \omega_{\mathbf{q}}, k_{0}^{i}=\frac{1}{2} q^{i}$. The higher excited states can be easily found to correspond to $k_{N}^{\mu}=\left(N+\frac{1}{2}\right) q^{\mu}$. They are generated by acting by the creation operator

$$
a^{\dagger}(\mathbf{q})=\frac{1}{\sqrt{2}}\left(-\sqrt{\varkappa} \partial_{y}+\frac{1}{\sqrt{\varkappa}} q_{\mu} \gamma^{\mu} y\right)
$$

on the ground state $(2.14)$.

\section{Canonical vs. Precanonical I: Hamilton-Jacobi equations}

A brief comparison of the above two approaches to field quantization seems to reveal a huge conceptual distance between the both: whereas one is based on the language of functionals and functional differential equations the second one uses only (Cliffordvalued) functions and partial differential equations. In view of the well established success of the canonical quantization approach this might mean a no-go sentence to the precanonical approach. This conclusion, however, appears to be too hasty. In this section we demonstrate how both approaches can be related to each other on the classical level of Hamilton-Jacobi theory. This consideration hints to the existence of a similar relation between precanonical and canonical approach on the quantum level and partially suggests a technique of establishing such a relation.

Various aspects of the interplay between the precanonical DW theory and the canonical Hamiltonian formalism have been discussed earlier [21, 27, 29, 33 35]. It has been shown that a general idea of relating the precanonical quantities and structures to the canonical ones is (i) to perform the space-time decomposition, (ii) to restrict the precanonical quantities to the Cauchy surface $\Sigma$ in the covariant configuration space, $\Sigma:(y=y(\mathbf{x}), t=$ const), and then (iii) integrate over it. 
Here we will show how the Hamilton-Jacobi equation of the De Donder-Weyl theory (DWHJ equation) [12 14

$$
\partial_{\mu} S^{\mu}+H\left(y^{a}, p_{a}^{\mu}=\frac{\partial S^{\mu}}{\partial y^{a}}\right)=0
$$

can be related to the functional differential Hamilton-Jacobi equation of the canonical formalism:

$$
\partial_{t} \mathbf{S}+\mathbf{H}\left(y(\mathbf{x}), \pi(\mathbf{x})=\frac{\delta \mathbf{S}}{\delta y(\mathbf{x})}\right)=0 .
$$

We basically shall argue that the following functional constructed from the DW eikonal functions $S^{\mu}\left(y^{a}, x^{\mu}\right)$

$$
\mathbf{S}:=\left.\int_{\Sigma}\left(S^{\mu} \omega_{\mu}\right)\right|_{\Sigma}=\left.\int_{\Sigma} S^{t}\right|_{\Sigma} d \mathbf{x}
$$

where $\left.\omega_{\mu}:=\partial_{\mu}\right\lrcorner\left(d x^{1} \wedge d x^{2} \wedge d x^{3} \wedge d x^{t}\right)$ and $\left.S^{t}\right|_{\Sigma}:=S^{t}\left(y^{a}=y^{a}(\mathbf{x}), \mathbf{x}, t\right)$ is a restriction of the time-like component of $S^{\mu}\left(y^{a}, x^{\mu}\right)$ 's to $\Sigma$, fulfills the standard functional differential HJ equation as a consequence of the partial differential DWHJ equation for $S^{\mu}$ 's.

For simplicity, let us consider the specific case of the scalar field. Then the DWHJ equation takes the form (see (2.8) and (3.1))

$$
\partial_{t} S^{t}+\partial_{i} S^{i}+\frac{1}{2} \partial_{y} S^{\mu} \partial_{y} S_{\mu}+\frac{1}{2} m^{2} y^{2}=0
$$

and the standard functional differential HJ equation reads (see (2.2) and (3.2))

$$
\partial_{t} \mathbf{S}+\frac{1}{2} \int d \mathbf{x}\left(\left(\frac{\delta \mathbf{S}}{\delta y(\mathbf{x})}\right)^{2}+\left(\partial_{i} y(\mathbf{x})\right)^{2}+m^{2} y(\mathbf{x})^{2}\right)=0 .
$$

From (3.3) we obtain

$$
\begin{aligned}
\partial_{t} \mathbf{S} & =\left.\int d \mathbf{x} \partial_{t} S^{t}\right|_{\Sigma}, \\
\frac{\delta \mathbf{S}}{\delta y(\mathbf{x})} & =\left.\partial_{y} S^{t}\right|_{\Sigma} .
\end{aligned}
$$

The equation for $\left.S^{\mu}\right|_{\Sigma}$ can be obtained from the DWHJ equation by noticing that when acting on $\left.S^{\mu}\right|_{\Sigma}$ the spatial derivative $\partial_{i}$ turns into the total derivative $\frac{d}{d x^{i}}:=\partial_{i}+\partial_{i} y(\mathbf{x}) \partial_{y}$ the last term of which should be compensated. Therefore, the equation for $\left.S^{\mu}\right|_{\Sigma}$ assumes the form (in the metric signature ---+ )

$$
\left.\partial_{t} S^{t}\right|_{\Sigma}+\left.\frac{d}{d x^{i}} S^{i}\right|_{\Sigma}-\left.\partial_{i} y(\mathbf{x}) \partial_{y} S^{i}\right|_{\Sigma}+\frac{1}{2}\left(\left.\partial_{y} S^{t}\right|_{\Sigma}\right)^{2}-\frac{1}{2}\left(\left.\partial_{y} S^{i}\right|_{\Sigma}\right)^{2}+\frac{1}{2} m^{2} y(\mathbf{x})^{2}=0
$$

Substituting $\left.\partial_{t} S^{t}\right|_{\Sigma}$ from this equation into the right hand side of (3.6) and using (3.7) we obtain

$$
\partial_{t} \mathbf{S}+\int d \mathbf{x}\left(\frac{1}{2}\left(\frac{\delta \mathbf{S}}{\delta y(\mathbf{x})}\right)^{2}+\left.\frac{d}{d x^{i}} S^{i}\right|_{\Sigma}-\left.\partial_{i} y(\mathbf{x}) \partial_{y} S^{i}\right|_{\Sigma}-\frac{1}{2}\left(\left.\partial_{y} S^{i}\right|_{\Sigma}\right)^{2}+\frac{1}{2} m^{2} y(\mathbf{x})^{2}\right)=0
$$


The second term under the integral does not contribute being a total divergence. The third and the forth terms together lead to $\frac{1}{2}\left(\partial_{i} y(\mathbf{x})\right)^{2}$ because in the DWHJ theory $\partial_{y} S^{i}=p^{i}$ and, for the scalar field, $\left.p^{i}\right|_{\Sigma}=-\partial_{i} y(\mathbf{x})$. We, therefore, obtained the functional differential HJ equation, eq. (3.5), as a consequence of the DWHJ equation (3.4) restricted to the Cauchy surface $\Sigma$ and a natural hypothesis (3.3) as to how the Hamilton-Jacobi eikonal functional $\mathbf{S}$ is related to the DWHJ eikonal functions $S^{\mu}$.

Similar idea of the restriction of a precanonical equation to the Cauchy surface $\Sigma$ will be used below to clarify the interrelation between the functional Schrödinger equation and our proposed Dirac-like generalized Schrödinger equation for quantized fields. Since the Hamilton-Jacobi equations (3.4) and (3.5) correspond to the classical limit of the Schrödinger equations (1.2), (2.11) and (2.4), (2.5), respectively, this interrelation appears now to be quite natural.

\section{Canonical vs. Precanonical II: wave function(al)s and wave equations}

As we have mentioned above, the wave function $\Psi\left(y^{a}, x^{\mu}\right)$ in eq. (1.2) is the probability amplitude of finding the values $y^{a}$ of the fields in the space-time point $x^{\mu}$. It is natural to ask how this quantity could be related to the Schrödinger wave functional $\boldsymbol{\Psi}_{S}\left(\left[y^{a}(\mathbf{x})\right], t\right)$ which is known to be the probability amplitude of observing the field configuration $y^{a}(\mathbf{x})$ on a space-like hypersurface of constant time $t$. A related question is how the functional differential equation for the Schrödinger wave functional $\boldsymbol{\Psi}_{S}\left(\left[y^{a}(\mathbf{x})\right], t\right)$ is related to the Dirac-like partial differential equation for the function $\Psi\left(y^{a}, x^{\mu}\right)$.

An obvious idea is to view the probability amplitude of observing a configuration $y^{a}(\mathbf{x})$ as a composition of single amplitudes of observing the corresponging values of the field $y^{a}=y^{a}(\mathbf{x})$ at each point $\mathbf{x}$. For this purpose we restrict the wave function $\Psi\left(y^{a}, x^{\mu}\right)$ to a Cauchy surface $\Sigma: t=$ const, $y^{a}=y^{a}(\mathbf{x})$, to obtain the function $\left.\Psi\right|_{\Sigma}:=$ $\Psi\left(y^{a}(\mathbf{x}), \mathbf{x}, t\right)$ which is a probability amplitude of observing the specific value $y^{a}(\mathbf{x})$ of the field in the given spatial point $\mathbf{x}$ (at the moment of time $t$ ), and then construct a joint probability amplitude of simultaneously observing the respective values $y^{a}=y^{a}(\mathbf{x})$ at all points $\mathbf{x}$ of an equal-time hypersurface with the time label $t$. This amplitude, to be denoted $\boldsymbol{\Psi}\left(\left[y^{a}(\mathbf{x})\right], t\right)$, then describes the same as the Schrödinger wave functional does.

If there are no correlations between the amplitudes $\Psi\left(y^{a}(\mathbf{x}), \mathbf{x}, t\right)$ at space-like separated points the composed amplitude $\boldsymbol{\Psi}\left(\left[y^{a}(\mathbf{x})\right], t\right)$ can be represented as the product of the single amplitudes given by $\left.\Psi\right|_{\Sigma}$ over all spatial points $\mathbf{x}$, i.e.

$$
\Psi\left(\left[y^{a}(\mathbf{x})\right], t\right)=\left.\prod_{\mathbf{x} \in \Sigma} \Psi\right|_{\Sigma}=\prod_{\mathbf{x}} \Psi\left(y^{a}(\mathbf{x}), \mathbf{x}, t\right) .
$$

The above continual product expression can be interpreted as follows:

$$
\prod_{\mathbf{x}} \Psi\left(y^{a}(\mathbf{x}), \mathbf{x}, t\right)=e^{\sum_{\mathbf{x}} \ln \Psi\left(y^{a}(\mathbf{x}), \mathbf{x}, t\right)}=\lim _{\Delta x \rightarrow 0} e^{\frac{1}{(\Delta x)^{3}} \int d \mathbf{x} \ln \Psi\left(y^{a}(\mathbf{x}), \mathbf{x}, t\right)},
$$

if the ordering problem of the matrix valued $\left.\Psi\right|_{\Sigma}$ 's at different points $\mathbf{x}$ and the problem of the existence of the limit are ignored. This symbolic formula motivates the following 
well-defined expression for the wave functional which is a quintessence of the above physical idea:

$$
\boldsymbol{\Psi}\left(\left[y^{a}(\mathbf{x})\right], t\right)=\operatorname{tr}\left((1+\beta) e^{\varkappa \int d \mathbf{x} \ln \Psi\left(y^{a}(\mathbf{x}), \mathbf{x}, t\right)}\right) .
$$

In (4.3) the trace serves to produce a scalar functional from the matrix valued one in (4.2), the ("very large") constant $\varkappa$, according to its physical meaning of the ultraviolet cutoff in the $\mathbf{k}$-space, replaces $1 /(\Delta x)^{3}$ in (4.2), and the projector to the posititive frequency part, $(1+\beta)$, is included under the trace for the reasons which will be shortly clarified.

Let us find now a functional differential equation satisfied by the functional (4.3) as a consequence of equation (2.12) for the wave function $\Psi\left(y^{a}, x^{\mu}\right)$ or, more precisely, of the corresponding equation for its restriction to the Cauchy surface $\Sigma,\left.\Psi\right|_{\Sigma}:=$ $\Psi\left(y^{a}(\mathbf{x}), \mathbf{x}, t\right)$. This equation is derived from (2.12) by the procedure which is identical to the one of deriving of equation (3.8) for $\left.S^{\mu}\right|_{\Sigma}$ from the DWHJ equation for $S^{\mu}$. It yields:

$$
\left.i \partial_{t} \Psi\right|_{\Sigma}=-\left.i \alpha^{i} \frac{d}{d x^{i}} \Psi\right|_{\Sigma}+\left.i \alpha^{i} \partial_{i} y^{a}(\mathbf{x}) \frac{\partial}{\partial y^{a}} \Psi\right|_{\Sigma}+\left.\frac{1}{\varkappa} \beta \widehat{H} \Psi\right|_{\Sigma}
$$

Now, for the time derivative of the functional $\boldsymbol{\Psi}([y(\mathbf{x})], t)$ given by (4.3) we obtain

$$
i \partial_{t} \Psi([y(\mathbf{x})], t)=\operatorname{tr}\left\{(1+\beta) e^{\varkappa \int d \mathbf{x} \ln \Psi} \varkappa \int d \mathbf{x} \Psi^{-1} i \partial_{t} \Psi\right\},
$$

where here and throughout the following calculation $\Psi$ denotes $\left.\Psi\right|_{\Sigma}=\Psi(y(\mathbf{x}), \mathbf{x}, t)$ and the consideration is confined, for simplicity, to the case of the one-component scalar field $y$. In what follows we also assume that $\Psi$ is an invertible matrix, though this assumption can be avoided by setting $\Psi=e^{W}$ and working dirctly in terms of $W$ instead of $\ln \Psi$.

Further,

$$
\frac{\delta \Psi}{\delta y(\mathbf{x})}=\operatorname{tr}\left\{(1+\beta) e^{\varkappa \int d \mathbf{x} \ln \Psi} \varkappa \Psi^{-1} \partial_{y} \Psi\right\}
$$

and

$$
\begin{aligned}
\frac{\delta^{2} \Psi}{\delta y(\mathbf{x})^{2}}= & \operatorname{tr}\left\{( 1 + \beta ) e ^ { \varkappa \int d \mathbf { x } \operatorname { l n } \Psi } \left(\varkappa^{2} \Psi^{-1} \partial_{y} \Psi \Psi^{-1} \partial_{y} \Psi-\varkappa \delta(0) \Psi^{-1} \partial_{y} \Psi \Psi^{-1} \partial_{y} \Psi\right.\right. \\
& \left.\left.+\varkappa \delta(0) \Psi^{-1} \partial_{y y}^{2} \Psi\right)\right\} .
\end{aligned}
$$

As usual, the second functional derivative at equal points is not well-defined because of the terms involving $\delta(\mathbf{x}=0)$ present. It requires a regularization which essentially amounts to replacing $\delta(0)$ with the momentum space cutoff. However, the latter already has its counterpart in our theory as the constant $\varkappa$. Under the regularization which identifies $\delta(0)$ with $\varkappa$ the first two terms in (4.7) cancel each other and we obtain

$$
\frac{\delta^{2} \Psi}{\delta y(\mathbf{x})^{2}}=\operatorname{tr}\left\{(1+\beta) e^{\varkappa \int d \mathbf{x} \ln \Psi} \varkappa^{2} \Psi^{-1} \partial_{y y}^{2} \Psi\right\}
$$


Now, the substitution of (4.4) into the right hand side of (4.5) yields

$$
i \partial_{t} \Psi=\operatorname{tr}\left\{(1+\beta) e^{\varkappa \int d \mathbf{x} \ln \Psi} \int d \mathbf{x} \Psi^{-1}\left(-i \varkappa \alpha^{i} \frac{d}{d x^{i}} \Psi+i \varkappa \alpha^{i} \partial_{i} y(\mathbf{x}) \partial_{y} \Psi+\beta \widehat{H} \Psi\right)\right\} .
$$

The first term under the integral reduces to the total divergence $\frac{d}{d x^{i}}\left(\alpha^{i} \ln \Psi\right)$ and does not contribute. The remaining terms lead to

$$
i \partial_{t} \Psi=\operatorname{tr}\left\{(1+\beta) e^{\varkappa \int d \mathbf{x} \ln \Psi} \int d \mathbf{x} \Psi^{-1}\left(i \varkappa \gamma^{i} \partial_{i} y(\mathbf{x}) \partial_{y} \Psi+\widehat{H} \Psi\right)\right\},
$$

where it is used that $(1+\beta) \beta=(1+\beta), \beta^{2}=1$. This is where the projection to the positive frequency part in (4.3) is crucial for the argument.

Substituting (2.11) to (4.9) and comparing the result with (4.8) we obtain

$$
\begin{aligned}
i \partial_{t} \Psi([y(\mathbf{x})], t)= & \frac{1}{2} \int d \mathbf{x}\left(-\frac{\delta^{2}}{\delta^{2} y(\mathbf{x})}+m^{2} y^{2}(\mathbf{x})\right) \Psi([y(\mathbf{x})], t) \\
& +\operatorname{tr}\left\{(1+\beta) e^{\varkappa \int d \mathbf{x}^{\prime} \ln \Psi} \int d \mathbf{x} i \varkappa \partial_{i} y(\mathbf{x}) \gamma^{i} \Psi^{-1} \partial_{y} \Psi\right\} .
\end{aligned}
$$

The first two terms in (4.10) are identical to the corresponding terms in the functional differential Schrödinger equation, eqs. (2.4), (2.5). Let us turn to the last term which, with the aid of (4.6), can be written in the form

$$
\operatorname{tr}\left\{\int d \mathbf{x} \partial_{i} y(\mathbf{x}) i \gamma^{i} \frac{\delta}{\delta y(\mathbf{x})}\|\mathbf{\Psi}\|\right\}
$$

where $\|\Psi\|$ is the matrix-valued functional given by

$$
\|\Psi\|:=(1+\beta) e^{\varkappa \int d \mathbf{x} \ln \Psi(y(\mathbf{x}), \mathbf{x}, t)},
$$

such that $\Psi=\operatorname{tr}(\|\Psi\|)$.

In order to interpret this term let us notice that the canonical Hamiltonian density $\mathcal{H}=T_{t}^{t}:=p^{t}(\mathbf{x}) \partial_{t} y(\mathbf{x})-L$ can be represented in the form (c. f. the definition of $H$ in $(1.1))$

$$
\mathcal{H}(\mathbf{x})=\left.H\right|_{\Sigma}(\mathbf{x})-\left.\partial_{i} y(\mathbf{x}) p^{i}\right|_{\Sigma}(\mathbf{x}),
$$

where $\left.H\right|_{\Sigma}(\mathbf{x})$ and $\left.p^{i}\right|_{\Sigma}(\mathbf{x})$ denote the quantities of the DW theory, the DW Hamiltonian $H$ and the spatial polymomenta $p^{i}$, restricted to the Cauchy surface $\Sigma$. We know that the operators of space-like polymomenta $p^{i}$ in the precanonical scheme are given by $-i \varkappa \gamma^{i} \partial_{y}$ (c. f. eq. (2.10)). Then (4.6) suggests that their restriction to $\Sigma$, the operators of $p^{i}(\mathbf{x})$, act on the functionals (4.3) as follows:

$$
\operatorname{tr}\left\{\hat{p}^{i}(\mathbf{x})\|\Psi\|\right\}=-\operatorname{tr}\left\{i \gamma^{i} \frac{\delta}{\delta y(\mathbf{x})}\|\Psi\|\right\} .
$$


Therefore, the expression in (4.11) is naturally identified with

$$
-\operatorname{tr}\left\{\int d \mathbf{x} \partial_{i} y(\mathbf{x}) \hat{p}^{i}(\mathbf{x})\|\Psi\|\right\}
$$

where the second term in (4.13) can be recognized. Consequently, eq. (4.10) can be viewed as the trace of the matrix functional differential Schrödinger equation

$$
i \partial_{t}\|\Psi\|-\widehat{\mathbf{H}}\|\boldsymbol{\Psi}\|=0
$$

where $\widehat{\mathbf{H}}$ is the matrix-valued functional differential operator corresponding to the Hamiltonian density written in the form (4.13):

$$
\widehat{\mathbf{H}}=\int d \mathbf{x} \widehat{\mathcal{H}}(\mathbf{x})=\int d \mathbf{x}\left(-\frac{1}{2} \frac{\delta^{2}}{\delta^{2} y(\mathbf{x})}+\frac{1}{2} m^{2} y^{2}(\mathbf{x})+i \partial_{i} y(\mathbf{x}) \gamma^{i} \frac{\delta}{\delta y(\mathbf{x})}\right) .
$$

Obviously, one would like to understand in greater details a relation between the Hamiltonian operator in (4.17) and the standard Schrödinger picture Hamiltonian operator $\widehat{\mathbf{H}}_{S}$, eq. (2.5). One can immediately ask if there exists a unitary transformation which relates $(4.17)$ to $(2.5)$ :

$$
\widehat{\mathbf{H}}_{S}=e^{-i \mathbf{N}} \widehat{\mathbf{H}} e^{i \mathbf{N}}
$$

where $\mathbf{N}$ is a functional operator to be found. A straightforward calculation yields

$$
\begin{aligned}
e^{-i \mathbf{N}} \widehat{\mathbf{H}} e^{i \mathbf{N}} & =\frac{1}{2} \int d \mathbf{x}\left(-\frac{\delta^{2} i \mathbf{N}}{\delta^{2} y(\mathbf{x})}-\left(\frac{\delta i \mathbf{N}}{\delta y(\mathbf{x})}\right)^{2}-2 \frac{\delta i \mathbf{N}}{\delta y(\mathbf{x})} \frac{\delta}{\delta y(\mathbf{x})}-\frac{\delta^{2}}{\delta^{2} y(\mathbf{x})}\right. \\
& \left.+2 i e^{-i \mathbf{N}} \partial_{i} y(\mathbf{x}) \gamma^{i} e^{i \mathbf{N}}\left(\frac{\delta i \mathbf{N}}{\delta y(\mathbf{x})}+\frac{\delta}{\delta y(\mathbf{x})}\right)+m^{2} e^{-i \mathbf{N}} y^{2}(\mathbf{x}) e^{i \mathbf{N}}\right)
\end{aligned}
$$

In order to obtain $\mathbf{H}_{S}$ in the right hand side of (4.19), we have to ensure first that the terms proportional to $\frac{\delta}{\delta y(\mathbf{x})}$ compensate each other. This requirement leads to the equation

$$
\frac{\delta \mathbf{N}}{\delta y(\mathbf{x})}=\gamma^{i} \partial_{i} y(\mathbf{x})
$$

Then $\frac{\delta^{2} \mathbf{N}}{\delta^{2} y(\mathbf{x})}=\gamma^{i} \partial_{i} \delta(0)=0$ and the other two terms in (4.19) yield

$$
\begin{aligned}
\left(\frac{\delta i \mathbf{N}}{\delta y(\mathbf{x})}\right)^{2} & =\left(\partial_{i} y(\mathbf{x})\right)^{2} \\
2 i e^{-i \mathbf{N}} \partial_{i} y(\mathbf{x}) \gamma^{i} e^{i \mathbf{N}} \frac{\delta i \mathbf{N}}{\delta y(\mathbf{x})} & =2\left(\partial_{i} y(\mathbf{x})\right)^{2}
\end{aligned}
$$

which are consistent with each other and with (4.20). The solution to (4.20) can be written in the form

$$
\mathbf{N}=\mathbf{N}[y(\mathbf{x}), \Sigma]=\int_{\Sigma} y(\mathbf{x}) \gamma^{i} d \Sigma_{i}
$$


where $\left.d \Sigma_{i}:=d y \wedge \partial_{i}\right\lrcorner\left(d x^{1} \wedge d x^{2} \wedge d x^{3}\right)$ (note, that $\Sigma$ is not varied under $\left.\frac{\delta}{\delta y(\mathbf{x})}\right)$. Obviously, the unitary tranformation generated by $\mathbf{N}$ leaves the $m^{2} y^{2}(\mathbf{x})$ term in $(4.17)$ invariant. Hence, summing up the results we obtain

$$
e^{-i \mathbf{N}} \widehat{\mathbf{H}} e^{i \mathbf{N}}=\frac{1}{2} \int d \mathbf{x}\left(-\frac{\delta^{2}}{\delta^{2} y(\mathbf{x})}+\left(\partial_{i} y(\mathbf{x})\right)^{2}+m^{2} y^{2}(\mathbf{x})\right)=\widehat{\mathbf{H}}_{S} .
$$

Thus, it is proven that the matrix-valued Hamiltonian operator (4.17) can be unitarily transformed to the standard Hamiltonian operator in the standard Schrödinger representation, eq. (2.5). Correspondingly, from (4.10) and (4.16) it follows that the wave functional

$$
\boldsymbol{\Psi}_{S}=\operatorname{tr}\left(e^{-i \mathbf{N}}\|\boldsymbol{\Psi}\|\right)
$$

fulfills the standard functional differential Schrödinger equation, eqs. (2.4) and (2.5). It is, therefore, the wave functional in the Schrödinger representation. Let us stress that the present results follow from the formula (4.3) constructing a wave functional $\Psi([y(\mathbf{x})], t)$ from the Clifford-valued wave function $\Psi\left(y^{a}, x^{\mu}\right)$ and the Dirac-like covariant generalized Schrödinger equation for the latter.

\subsection{An example: the vacuum state wave functional}

Let us demonstrate how the formula (4.3) can be used to construct the vacuum state wave functional, eq. (2.6), from the ground state wave function of the precanonical approach, eq. (2.14). The latter yields $\ln \Psi_{0}(y, \mathbf{q})=-\frac{1}{2 \varkappa} q^{\mu} \gamma_{\mu} y^{2}$. The restriction $\left.\left(\ln \Psi_{0}\right)\right|_{\Sigma}(\mathbf{x})$ in constructed by taking into account that

$$
\left.y\right|_{\Sigma}(\mathbf{x})=y(\mathbf{x})=\int \frac{d \mathbf{q}}{(2 \pi)^{3}} e^{i \mathbf{q x}} y(\mathbf{q})
$$

and assuming that

$$
\left.\left(q^{\mu} \gamma_{\mu} y\right)\right|_{\Sigma}(\mathbf{x}):=\int \frac{d \mathbf{q}}{(2 \pi)^{3}} e^{i \mathbf{q x}} q^{\mu} \gamma_{\mu} y(\mathbf{q})
$$

This allows us to write

$$
\begin{aligned}
\left.\int d \mathbf{x}\left(\ln \Psi_{0}\right)\right|_{\Sigma} & =-\frac{1}{2 \varkappa} \int d \mathbf{x} \int \frac{d \mathbf{q}}{(2 \pi)^{3}} e^{i \mathbf{q} \mathbf{x}} q^{\mu} \gamma_{\mu} y(\mathbf{q}) \int \frac{d \mathbf{q}^{\prime}}{(2 \pi)^{3}} e^{i \mathbf{q}^{\prime} \mathbf{x}} y\left(\mathbf{q}^{\prime}\right) \\
& =-\frac{1}{2 \varkappa} \int \frac{d \mathbf{q}}{(2 \pi)^{3}} \beta \omega_{\mathbf{q}} y(-\mathbf{q}) y(\mathbf{q}) .
\end{aligned}
$$

Substituting this result to (4.3) we obtain the standard vacuum state wave functional for the quantum scalar field, eq. (2.6):

$$
\Psi_{\mathrm{vac}}=\exp \left(-\frac{1}{2} \int \frac{d \mathbf{q}}{(2 \pi)^{3}} \omega_{\mathbf{q}} y(-\mathbf{q}) y(\mathbf{q})\right)
$$

as a result of the specific composition of the Clifford algebra valued ground state wave functions (2.14) as given by the formula (4.3). 


\section{Conclusions}

In this paper we have demonstrated how the standard functional Schrödinger representation in quantum field theory can be derived from the recently proposed precanonical field quantization which is based on a finite dimensional manifestly covariant generalization of the Hamiltonian formalism to field theory and leads to a covariant Dirac-like "multi-temporal" generalization of the Schrödinger equation on a finite dimensional configuration bundle of field variables over the space-time. Note that those are sections of this bundle which are the field configurations whose space is infinite dimensional and underlies the conventional canonical quantization.

We argued that the Schrödinger wave functional appears to be related to the continual product, over all spatial points, of the values of the wave function fulfilling the above Dirac-like equation restricted to a Cauchy data surface.2 In this sense the information contained in the Schrödinger wave functional can be extracted from the abovementioned wave function. In doing so the constant $\varkappa$ appearing in precanonical quantization is interpreted as a quantity of the ultraviolet cutoff scale.

The existence of the connection between the canonical and precanonical approaches to field quantization is an additional argument in favor of precanonical quantization, even though the latter is yet to be developed to the level of a calculational technique in quantum field theory. This connection is not restricted to the simple case of a free scalar field treated here. In fact, it can be straightforwadly extended to the interacting scalar fields. Moreover, a preliminary consideration shows that it is also possible to extend the argument to other fields, such as the Yang-Mills or the spinor fields. However, the technical difficulty we face then is that those fields are singular with respect to the De Donder-Weyl Legendre transformation $y_{\mu}^{a} \rightarrow p_{a}^{\mu}, L \rightarrow H$ and, therefore, they require a properly modified precanonical quantization procedure, similarly to the quantization of constrained systems. This work is in progress.

Note that the common infinities of the canonical quantization approach, such as the vacuum energy or the singularity in the second functional derivative in the Hamiltonian operator, are not present in the precanonical approach. Our discussion clearly indicates that these infinities appear only when the functional objects are constructed from the well defined quantities of the precanonical approach. However, not all infinities in quantum field theory are likely to be of this nature because the characteristic singularities of Green functions are persistent also in the precanonical approach.

Note also that the equations of the precanonical approach remain valid in the spacetimes which do not admit the space-time decomposition, i.e. are not globally hyperbolic, and, therefore, do not enable us to introduce the functional Schrödinger representation in a straightforward way. In this sense precanonical quantization seems to have a wider range of validity than canonical quantization, though its physical interpretation beyond the framework of globally hyperbolic space-times may require new physical insights.

${ }^{2}$ An earlier discussion of a possible continual product structure of the Schrödinger wave functional can be found in [1, 3, 36] (c. f. [37] for a related brief discussion but a conclusion different from ours). Such a structure is also characteristic to the ultralocal models extensively studied by Klauder 38. 


\section{Acknowledgements}

I am grateful to E. Gozzi, M. C. Muños-Lecanda, N. Roman-Roy, H. Römer, and F. Hélein for their kind hospitality during my visits to their departments which were stimulating for the present work. I also thank J. Klauder, for enlightening conversations on ulralocal models and for making the book [38] available to me prior to publication, and V. Tapia, for useful discussion and for bringing Ref. [36] to my attention. I am indebted to Prof. A. Wipf and the Institute of Theoretical Physics in Jena for their kind hospitality and the opportunity to pursue this research.

\section{References}

[1] I.V. Kanatchikov, Toward the Born-Weyl quantization of fields, Int. J. Theor. Phys. 37 (1998) 333-42, Preprint quant-ph/9712058.

[2] I.V. Kanatchikov, De Donder-Weyl theory and a hypercomplex extension of quantum mechanics to field theory, Rep. Math. Phys. 43 (1999) 157-70, Preprint hep-th/9810165.

[3] I.V. Kanatchikov, On quantization of field theories in polymomentum variables, in: Particles, Fields and Gravitation (Proc. Int. Conf. devoted to the memory of Prof. R. Raczzka, Łodz, Poland, Apr. 1998) ed. J. Rembieliński, AIP Conf. Proc. vol. 453 (1998) 356-67, Preprint hep-th/9811016.

[4] I.V. Kanatchikov, From the De Donder-Weyl Hamiltonian formalism to quantization of gravity, in: Current Topics in Mathematical Cosmology (Proc. Int. Seminar, Potsdam, Germany, Mar 30-Apr 04 1998) eds. M. Rainer and H.-J. Schmidt (Singapore: World Scientific) 457-67, Preprint gr-qc/9810076.

[5] I.V. Kanatchikov, Towards a "pre-canonical" quantization of gravity without the space+time decomposition, Preprint FSU TPI 01/99, gr-qc/9909032.

[6] I.V. Kanatchikov, Quantization of gravity: yet another way, in: Coherent States, Quantization and Gravity (Proc. 17th Workshop on Geometric Methods in Physics (WGMP 98), Białowieża, Poland, 3-9 July 1998) in press, Preprint gr-qc/9912094.

[7] I.V. Kanatchikov, Precanonical perspective in quantum gravity, Nucl. Phys. B Proc. Suppl. 88 (2000) 326-330, Preprint gr-qc/0004066.

[8] I.V. Kanatchikov, Precanonical Quantum Gravity: quantization without the space-time decomposition, to appear in Int. J. Theor. Phys. 40 (2001) No. 6, Preprint gr-qc/0012074.

[9] I.V. Kanatchikov, Covariant geometric prequantization of fields, contributed paper to the Proc. Ninth Marcel Grossmann Meeting, Rome (Italy) July 2000 (to be published by World Scientific, Singapore), Preprint gr-qc/0012038.

[10] Th. De Donder, Theorie Invariantive du Calcul des Variations, Gauthier-Villars, Paris (1935).

[11] H. Weyl, Geodesic fields in the calculus of variations, Ann. Math. (2) 36, 607-29 (1935).

[12] H. Rund, The Hamilton-Jacobi Theory in the Calculus of Variations, D. van Nostrand, Toronto (1966).

[13] H. Kastrup, Canonical theories of Lagrangian dynamical systems in physics, Phys. Rep. 101 (1983) 1-167. 
[14] J. von Rieth, The Hamilton-Jacobi theory of De Donder and Weyl applied to some relativistic field theories, J. Math. Phys. 25 (1984) 1102-15.

[15] Th. H. J. Lepage, Acad. Roy. Belgique Bull. Cl. Sci. (5e Sér), 27 (1941) 27.

[16] Th. H. J. Lepage, ibid., 28 (1942) 73, 247.

[17] P. Dedecker, On the generalizations of symplectic geometry to multiple integrals in the calculus of variations, Lect. Notes Math. vol. 570 (Berlin: Springer, 1977) 395-456

[18] D. Krupka, Lepagean forms in high order variational theory, in: Proc IUTAM-ISIMM Symp on Modern Developments in Analytical Mechanics vol. 1, Atti Accad. Sci. Torino Suppl. 117 (1983) 197-238.

[19] D. Krupka, Geometry of Lagrangean structures. 3 Suppl. Rend. Circ. Mat. Palermo. Ser II No 14 (1987) 187-224.

[20] M.J. Gotay, An exterior differential systems approach to the Cartan form, in: Symplectic Geometry and Mathematical Physics, eds. Donato P et al (Boston: Birkhäuser, 1991) 160-88

[21] I.V. Kanatchikov, Canonical structure of classical field theory in the polymomentum phase space, Rep. Math. Phys. 41 (1998) 49-90, Preprint hep-th/9709229.

[22] I.V. Kanatchikov, On field theoretic generalizations of a Poisson algebra, Rep. Math. Phys. 40 (1997) 225-234, Preprint hep-th/9710069.

[23] I.V. Kanatchikov, Novel algebraic structures from the polysymplectic form in field theory in: GROUP21, Physical Applications and Mathematical Aspects of Geometry, Groups and Algebras vol. 2, eds. Doebner H-D et al (Singapore: World Scientific) 894-9, Preprint hep-th/9612255.

[24] C. Paufler, A vertical exterior derivative in multisymplectic geometry and a graded Poisson bracket for nontrivial geometries, to appear in Rep. Math. Phys. 47 (2001), Preprint math-ph/0002032.

[25] C. Paufler, On the geometry of field theoretic Gerstenhaber structures, the talk at the XXXII Symp. on Math. Phys. (Torun, Poland) June 2000. Preprint math-ph/0102012.

[26] M. Forger and H. Römer, A Poisson Bracket on Multisymplectic Phase Space, to appear in Rep. Math. Phys. (2001), Preprint math-ph/0009037.

[27] F. Hélein and J. Kouneiher, Finite dimensional Hamiltonian formalism for gauge and field theories, Preprint math-ph/0010036.

[28] M.J. Gotay, J. Isenberg, J. Marsden, Momentum Maps and Classical Relativistic Fields (Berkeley preprint, various versions exist since 1985), Part I: Covariant Field Theory, Preprint physics/9801019; Part II: Canonical Analysis of Field Theories, in progress.

[29] J.E. Nelson and T. Regge, Covariant canonical formalism for gravity, Ann. Phys. 166 (1986) 234.

[30] B. Hatfield, Quantum Field Theory of Point Particles and Strings, Reading, MA: Addison-Wesley (1992).

[31] S.S. Schweber, "An Introduction to Relativistic Quantum Field Theory", Harper and Row, New York (1961).

[32] P. Mansfield, M. Sampaio and J. Pachos, Short distance properties from large distance behaviour, Int. J. Mod. Phys. A13 (1998) 4101-4122, Preprint hep-th/9702072 (see also the references cited therein). 
[33] M.J. Gotay, A multisymplectic framework for classical field theory and the calculus of variations I. Covariant Hamiltonian formalism, in: Mechanics, Analysis and Geometry: 200 Years after Lagrange ed. M. Francaviglia, Amsterdam: North Holland (1991) 203-35.

[34] M.J. Gotay, A multisymplectic framework for classical field theory and the calculus of variations II. Space + time decomposition, Diff. Geom. and its Appl. 1 (1991) 375-90

[35] J. Śniatycki, The Cauchy data space formulation of classical field theory, Rep. Math. Phys. 19 (1984) 407-22.

[36] V. Tapia, A novel method to solve functional differential equations: application to quantum field theory, Nuovo Cim. 105B (1990) 667-83.

[37] M. Navarro, Comments on Good's Proposal for New Rules of Quantization, J. Math. Phys. 36 (1995) 6665-6672, Preprint hep-th/9503068.

[38] J.R. Klauder, Beyond Conventional Quantization (Cambridge University Press, Cambridge 1999). 